\title{
Real-Time In Situ Secondary Structure Analysis of Protein Monolayer with Mid-Infrared Plasmonic Nanoantennas
}

\author{
Dordaneh Etezadi, ${ }^{\dagger}$ John B. Warner, IV, ${ }^{\ddagger}$ Ji Hilal A. Lashuel, ${ }^{\ddagger}$ and Hatice Altug ${ }^{*}{ }^{\dagger}$ \\ ${ }^{\dagger}$ Bionanophotonic Systems Laboratory and ${ }^{\ddagger}$ Laboratory of Molecular and Chemical Biology of Neurodegeneration, Brain Mind \\ Institute, Faculty of Life Sciences, École Polytechnique Fédérale de Lausanne (EPFL), Lausanne 1015, Switzerland
}

\section{Supporting Information}

\begin{abstract}
Dynamic detection of protein conformational changes at physiological conditions on a minute amount of samples is immensely important for understanding the structural determinants of protein function in health and disease and to develop assays and diagnostics for protein misfolding and protein aggregation diseases. Herein, we experimentally demonstrate the capabilities of a mid-infrared plasmonic biosensor for real-time and in situ protein secondary structure analysis in aqueous environment at nanoscale. We present label-free ultrasensitive dynamic monitoring of $\beta$-sheet to disordered conformational transitions in a monolayer of the disease-related $\alpha$-synuclein protein under varying stimulus conditions. Our experiments show that the extracted secondary structure signals from plasmonically enhanced amide I signatures in the protein monolayer can be reliably and reproducibly acquired with second derivative analysis for dynamic monitoring. Furthermore, by using a polymer layer we show that our nanoplasmonic approach of extracting the frequency components of vibrational signatures matches with the results attained from gold-standard infrared transmission measurements. By facilitating conformational analysis on small quantities of immobilized proteins in response to external stimuli such as drugs, our plasmonic biosensor could be used to introduce platforms for screening small molecule modulators of protein misfolding and aggregation.
\end{abstract}

KEYWORDS: plasmonic, nanoantennas, mid-infrared, protein secondary structure, real-time analysis

$I^{\text {natsen }}$ nnovative surface-based optical nanosensors offer novel means to investigate biomolecular processes in a nondestructive and label-free manner and thus render exceptional possibilities for biochemical analysis toward developing early detection and diagnostics. $^{1-3}$ In particular, nanoplasmonic sensors are attractive chip-based platforms that provide strongly confined surface plasmon excitations at the sensor interface to interact with thin layers of bioanalytes for highly sensitive detection at molecular dimensions. ${ }^{4-7}$ Besides their extensive applications at visible and near-infrared frequencies, engineered nanoplasmonic structures deliver unique possibilities for biomolecular studies with ultrasensitive surface-enhanced infrared absorption spectroscopy based on the distinct molecular vibrations in the mid-infrared (mid-IR) range. $^{8-12}$ The mid-IR vibrational signatures of molecules correspond to chemical bonds among their constituent elements and therefore enable nondestructive and label-free characterization. By providing orders of magnitude signal enhancement of these chemical fingerprints, engineered metallic nanostructures represent promising systems for sensitive detection of biomolecules and provide the means to overcome the limitations of conventional infrared absorption spectroscopy. ${ }^{13-15}$ Molecular information accessible with mid-IR spectroscopy is of great importance for analysis of numerous biomolecules such as lipid membranes and proteins, with significant implications in the biological and pharmaceutical industries. $^{16,17}$

As the main components of biological processes, in-depth investigations of proteins' properties and structures are imperative to elucidate their functions in health and disease. ${ }^{18,19}$ Mid-IR protein signatures incorporate comprehensive information on protein secondary structure in association with hydrogen bonding among their functional groups including carboxylic acid and amine groups that can be affected by environmental conditions. ${ }^{20-23}$ Developing sensitive tools that can provide insight into the dynamics and conformational properties of proteins is crucial for deciphering the molecular mechanisms underlying several systemic and neurodegenerative diseases caused by protein misfolding and aggregation. These neurodegenerative diseases include Alzheimer's (AD), Parkinson's (PD), and Huntington's (HD) diseases to name a few. ${ }^{24}$ Despite the fact that protein misfolding is widely accepted as the primary triggering event in the pathogenesis of such diseases, the structural changes associated with the misfolding, aggregation, and formation of the pathological hallmarks of the disease remain poorly understood. ${ }^{25}$ In general, the fibrillization

Received: February 5, 2018

Accepted: May 30, 2018

Published: May 30, 2018 
process linked to neurodegeneration follows a nucleationdependent mechanism that includes formation of partially folded intermediates of protein species. Nevertheless, their roles in disease development are subject to debate, in part due to limitations of conventional techniques in accessing dynamic conformational characteristics of such unstable proteins during misfolding events at physiological conditions. It is also noteworthy that current techniques including circular dichroism and conventional Fourier transform IR spectroscopy require large quantities of material and are not amenable to switching different solutions for dynamic analysis. ${ }^{21,26}$ Therefore, enabling label-free, ultrasensitive, and real-time monitoring of protein conformational transitions in response to different stimuli with minimum sample preparation can provide the means to study metastable folding/misfolding intermediates involved in diseases.

In this work, we present real-time detection and monitoring of secondary structural transitions in a protein monolayer in response to stimuli in aqueous solution enabled by mid-IR plasmonic nanoantennas. Our label-free biosensing platform constituted of gold nanoantenna arrays provides localized surface plasmon resonances in the mid-IR spectrum that are engineered for ultrasensitive detection of biomolecules in their vicinity through enhanced vibrational fingerprints even in the presence of water. We previously demonstrated the capabilities of this platform for reliable detection of secondary structural signatures from different proteins immobilized on nanoantennas at static conditions. ${ }^{27}$ Here, we demonstrate for the first time dynamic in situ secondary structure investigations on a protein monolayer by analyzing its plasmonically enhanced amide I fingerprint. As a model system, we employed the Parkinson's disease-related protein $\alpha$-synuclein ( $\alpha$-Syn). $\alpha$-Syn, an intrinsically disordered intracellular protein, is the primary component of intracellular $\beta$-sheet aggregates that are characteristics of several neurodegenerative disorders including dementia, PD, and Lewy Body dementia, also known as synucleinopathies. ${ }^{28}$ In addition to forming $\beta$-sheet-rich pathological fibrils, $\alpha$-Syn can also adopt $\alpha$-helical structures when bound to either vesicles or membranes. ${ }^{29,30}$ The $\beta$-sheet or $\alpha$-helical structures have previously been induced in this protein using organic solvents. ${ }^{31}$ In our experiments, we trigger dynamic conformational variations by modifying the environmental conditions and introducing stimulus to induce reversible secondary structural changes in the immobilized $\alpha$-Syn monolayer. The acquired amide I signature of the monolayer allows distinguishing transformations and variations of different secondary structures in the protein sample by decomposing this vibrational fingerprint to its constituting components. We demonstrate the disappearance and formation of $\beta$-sheet signatures upon titration of methanol $(\mathrm{MeOH})$ in real time. In addition, we show that exposing the immobilized $\alpha$-Syn to denaturing conditions restores the natively disordered structure in the protein monolayer. Our approach enables monitoring distinct $\beta$-sheet secondary structure changes in protein monolayers where partial folding transitions can be observed. Moreover, we experimentally extract the frequency of the spectral component from the vibrational signature of a polymer layer using our nanoplasmonic approach as well as infrared transmission and infrared reflection-absorption spectroscopy. Our comparison confirms the agreement of the extracted results for the frequency of the vibrational component from nanoplasmonic substrate to that of conventional IR transmission measurement technique. Thus, we establish the reliability of this approach as a new toolkit for mid-IR protein secondary structure analysis that can provide complementary information to those obtained through bulk measurements. Enabling dynamic monitoring of protein conformational changes with our sensor can be promising for studying protein secondary structure transitions of invaluable proteins or protein complexes isolated from biological samples that are obtained in minute quantities using multisteps laborious protocols (e.g., protein aggregates isolated from patients' brains or cellular and animal models of neurodegenerative disorders).

\section{EXPERIMENTAL SECTION}

Nanofabrication and Fluidic Device. Cleaned $\mathrm{CaF}_{2}$ substrates were spin coated with methyl methacrylate (MMA) and poly(methyl methacrylate) (PMMA) 495 MW resist double layer followed by electron beam exposure using Vistec EBPG5000 at $100 \mathrm{kV}$. Metal nanoantenna arrays of $100 \mu \mathrm{m} \times 100 \mu \mathrm{m}$ were formed through a liftoff process by evaporating a thin $\mathrm{Cr}$ adhesion layer and $100 \mathrm{~nm}$ of $\mathrm{Au}$ with a Leybold Optics LAB $600 \mathrm{H}$ e-beam evaporator. The fabricated plasmonic substrates were cleaned with $\mathrm{O}_{2}$ plasma treatment before use. A fluidic device constituted of a three-layer PDMS (polydimethylsiloxane) chamber is used to realize the fluidic channel, in/ outlet flow channels and top seal layer for bioexperiments. The nanoplasmonic substrate is loaded on the flow channel and the device is mounted on the IR microscope stage using a metallic clamp for sealing and measurements. Thin PMMA coating at different thicknesses on gold and nanoplasmonic surfaces is prepared by spin coating different dilutions of the polymer in anisole at $6000 \mathrm{rpm}$ and was calibrated using an ellipsometer (Sopra GES 5E) to be used for measurements in dry conditions.

Fourier Transform Infrared (FTIR) Measurements, Data Analysis, and Numerical Simulation. A Bruker Vertex-80 V interferometer equipped with a Globar infrared source and $\mathrm{KBr}$ beamsplitter $\left(4000-400 \mathrm{~cm}^{-1}\right)$, coupled to a Hyperion 3000 IR microscope with Cassegrain objective $(15 \times, \mathrm{NA}=0.4)$ and a liquid nitrogen cooled mercury cadmium telluride detector (MCT) was used for the IR measurements during the bioexperiments. The interferometer was kept under vacuum while the microscope, including the sample stage, was purged with $\mathrm{CO}_{2}$ and humidity filtered air. A linear polarizer along the long axis of the nanoantenna arrays was used on the incident light path. In the normal incident FTIR measurements the knife edge apertures at the microscope were aligned to the area of the arrays, i.e., $100 \mu \mathrm{m}$ by $100 \mu \mathrm{m}$. An Au mirror was used for reference measurement as background. All data acquisition was performed at resolution of $4 \mathrm{~cm}^{-1}$ and 1024 scans co-added for each spectrum which corresponds to $4 \mathrm{~min}$ acquisition time. Second derivative of the logarithmic ratio of reflectance responses $(A)$ were extracted using Savitzky-Golay smoothing with a polynomial fitting on the acquired data within the amide I range. Commercial software package of Lumerical FDTD Solutions was employed for field-profile illustration in Figure $1 \mathrm{~b}$. A plane wave source was used for plasmonic excitation of the array unit cell (L: $1850 \mathrm{~nm}, \mathrm{P}: 2600 \mathrm{~nm}$ ) with a linearly polarized electric field along the antenna length. The dielectric values of gold and water were from Palik library, whereas constant refractive index of $n=1.4$ was used for $\mathrm{CaF}_{2}$ substrate.

Protein Preparation and Surface Functionalization. $\alpha$-Syn monomeric samples were produced in E.Coli with C-terminal sulfhydryl groups (A140C) for immobilization via a PEG linker and were diluted in $50 \mathrm{mM}$ Tris $\mathrm{HCl}$ buffer with $150 \mathrm{mM} \mathrm{NaCl}$ (TBS) at $\mathrm{pH} 7.4$ in stock solution of $70 \mu \mathrm{M}$. Synthesized amino-(PEG) $)_{2}$ Maleimide linker were prepared as explained in ref 27 to provide a hydrophilic environment and high flexibility for the immobilized proteins. For Au surface functionalization and ex situ immobilization of protein, a solution of $0.5 \mathrm{mM}$ MHDA (16-Mercaptohexadecanoic acid, Sigma-Aldrich) in ethanol is used to form the self-assembledmonolayer (SAM) by overnight immersion, followed by activation of the carboxyl groups with a solution of $0.05 \mathrm{M} \mathrm{N}$-hydroxysulfosuccinimide (sulfo-NHS) (Thermo Fisher Scientific) and 0.2 M 1-ethyl-3- 
a)

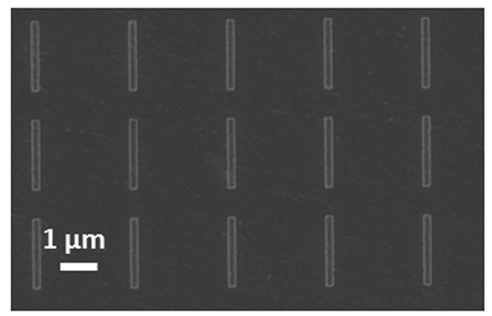

b)

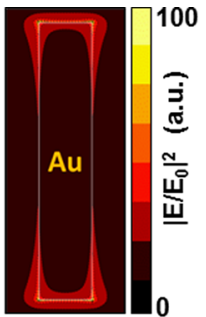

c)

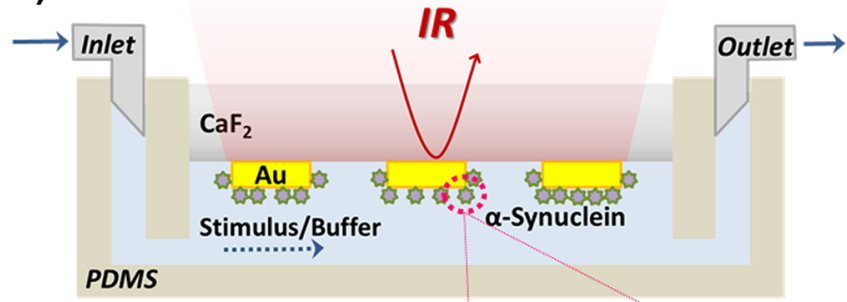

d)

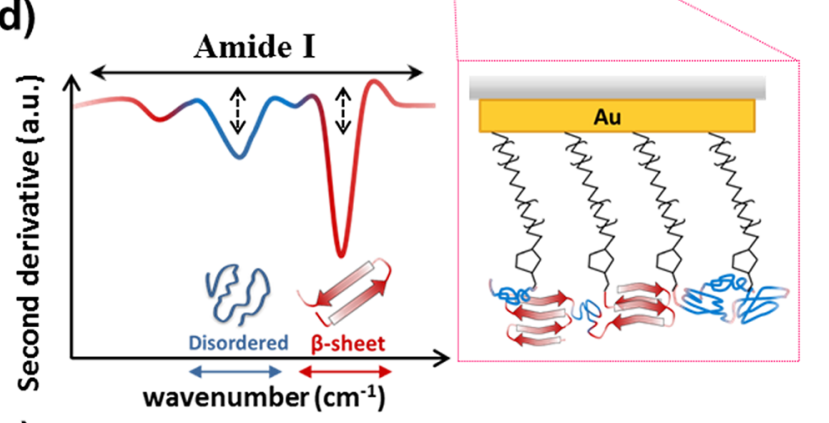

e)

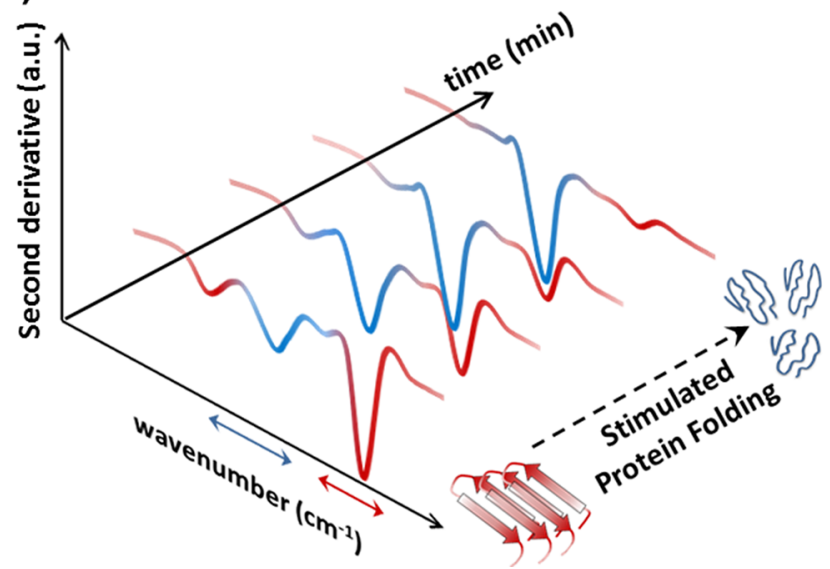

Figure 1. Experimental implementation for real-time in situ secondary structure monitoring of a protein monolayer using plasmonically enhanced amide I vibrational signature with mid-IR nanoantennas. (a) SEM image of a typical gold nanorod antenna array on $\mathrm{CaF}_{2}$ substrate. (b) Simulated field profile (intensity enhancement) at the top surface of an antenna at $1700 \mathrm{~cm}^{-1}$, produced with FDTD simulation for L: $1850 \mathrm{~nm}$ and P: $2600 \mathrm{~nm}$ in water. The excited hot-spots at antenna tip-ends are clearly observable. (c) Schematic illustration for integrated mid-IR biosensor with the fluidic device incorporating antenna arrays for real-time in situ measurements on immobilized protein monolayer in aqueous solution with plasmonic internal reflection configuration (not to scale). Different stimulus contents are introduced in the fluidic channel while the antenna array reflectance spectrum is measured at time intervals. (d) Representative diagram of the $\alpha$-Syn protein monolayer immobilized on antenna arrays (right) with different secondary structures (not to scale) and the corresponding conformational components in the spectrum of the amide I signature extracted with second derivative analysis (left). Red color corresponds to $\beta$-sheet
Figure 1. continued

components while blue represents random component (disordered conformation). (e) Monitoring secondary structural changes in immobilized protein monolayer in real time with the amide I conformational components upon introduction of stimuli. Gradual decrease of $\beta$-sheet signature and increase of non $\beta$-sheet signals (e.g., disordered) can be detected as a function of time.

(3-(dimethylamino)propyl) carbodiimide hydrochloride (EDC) (Thermo Fisher Scientific) in 0.1 M MES solution within $20 \mathrm{~min}$. After a water rinse step, $100 \mu \mathrm{L}$ of $12 \mu \mathrm{M}$ amino-(PEG) $)_{2}$-maleimide linker in TBS is incubated on all antenna arrays within $45 \mathrm{~min}$. Then, the substrate is rinsed with buffer and incubated with the protein. Therefore, the regions that undergo conformational transitions in the protein (residues 1-99) would remain exposed after immobilization. All experiments and biochemical preparations were conducted at room temperature.

\section{RESULTS AND DISCUSSION}

Real-Time Biosensing Principle. The plasmonic sensor is constituted of gold nanorod antenna arrays fabricated on IRtransparent $\mathrm{CaF}_{2}$ substrates using electron beam lithography. Figure la shows an SEM image of one of the arrays on the plasmonic substrate. The resonance peak tuning of mid-IR nanorods follows a linear relation with the antenna aspect ratio and the effective refractive index of their surrounding environment. Therefore, by designing the length $(\mathrm{L})$ of the antennas and their period (P) in the array the plasmonic resonance can be engineered for collective excitation to probe different vibrational signatures in mid-IR range when the antennas are illuminated by polarized light along their long axis. ${ }^{32,33}$ Accordingly, we design the antenna arrays for resonances close to $1600-1700 \mathrm{~cm}^{-1}$ in aqueous environment. ${ }^{20-22}$ Here, the width $(\mathrm{W})$ and height $(\mathrm{H})$ of antennas are kept at constant values of 200 and $100 \mathrm{~nm}$, respectively, while the size of each array is $100 \mu \mathrm{m}$ by $100 \mu \mathrm{m}$. Mid-IR plasmonic nanorod arrays can provide optimum resonance bandwidths for detecting the amide I signature encompassing protein conformational information. Their dimensions for operation in aqueous media are suitable for employing conventional nanofabrication techniques to reliably and reproducibly manufacture the nanoplasmonic substrates, with potentials for wafer-scale and low-cost production using emerging fabrication methods. ${ }^{27,34}$ Figure $1 \mathrm{~b}$ shows the antenna hot-spots associated with the plasmonic resonance excitation observed in the fieldprofile (intensity enhancement) at the top surface of an antenna in an array (L: $1850 \mathrm{~nm}$ and P: $2600 \mathrm{~nm}$ ) in water that is obtained with finite-difference time-domain (FDTD) simulation (at $1700 \mathrm{~cm}^{-1}$ ). In order to enable measurement in aqueous solution, the nanoplasmonic substrate is mounted on a microfluidic device and used in plasmonic internal reflection measurement configuration. ${ }^{14,35}$ In this scheme, the reflection response of the antenna array is measured from the backside of the IR-transparent $\mathrm{CaF}_{2}$ substrate as illustrated in Figure 1c, while the solution surrounding the antennas in the fluidic channel can be changed using a flow system with a syringe pump. The overlap of the excited plasmonic hotspots in the vicinity of the nanorod tip-ends with the immobilized protein monolayer, shown schematically in the right panel of Figure 1d, results in ultrasensitive surface enhanced IR absorption spectroscopic detection. The initial reflectance response of the antenna array $(R)$ and its reflectance after immobilizing protein $\left(R_{\text {with protein }}\right)$ are used to extract the 
vibrational signatures of the monolayer using the logarithmic ratio of the responses, $A=-\log \left(R_{\text {with protein }} / R_{\text {no protein }}\right)$, to ensure the compensation of the variations in the refractive index and absorption signals of the surrounding background. By means of second-derivative analysis on the acquired amide I signature of protein monolayer on the antenna array, we obtain information on its secondary structure composition through its constituting spectral components, as schematically depicted in the left panel of Figure $1 \mathrm{~d}$ where distinct $\beta$-sheet component can be differentiated from that of disordered conformation, helices or non- $\beta$ strands. ${ }^{20}$ In order to demonstrate dynamic sensing, secondary structure of $\alpha$-Syn monolayer immobilized on antennas is monitored in real time in different solutions. In this work, we are mainly monitoring the distinct strong $\beta$-sheet IR signals from $\alpha$-Syn monolayer that are detectable in its amide I component peaks at $\sim 1615-1640 \mathrm{~cm}^{-1}$ (red arrow in Figure 1d) and its disordered signals at $\sim 1645-1660 \mathrm{~cm}^{-1}$ (blue arrow in Figure 1d) overlapping with that of helices at different conditions. In addition, weaker $\beta$-sheet signatures at $\sim 1680-1695 \mathrm{~cm}^{-1}$ and that of non $\beta$-sheet strands (including turns and loops) at $\sim 1660-1690 \mathrm{~cm}^{-1}$ are also detected in the presented data and can be used for evaluation. We flow varying $\mathrm{MeOH}$ concentration in the buffer through the fluidic channel and detect the secondary structural variations of the immobilized protein in real time as illustrated in Figure 1e. While the conformational change is induced by introducing different concentration of $\mathrm{MeOH}$, the gradual variations between $\beta$-sheet and disordered signals of the amide I components can be detected dynamically.

Real-Time Secondary Structure Detection on Protein Monolayer. To demonstrate real-time secondary structure monitoring of a protein monolayer in aqueous solution, we investigated the secondary changes on $\alpha$-Syn protein in interaction with $\mathrm{MeOH}$. Stimulating secondary structure transitions in $\alpha$-Syn monolayer is achieved by introduction and removal of $\mathrm{MeOH}$ (Figure 1c-e), which can induce the formation of distinguishable $\beta$-sheet conformation at high percentage (e.g., 40\%) of $\mathrm{MeOH}$ compared to the native intrinsically disordered structure (see bulk CD measurement results in Figure $\mathrm{S} 1$ and Transmission Electron Microscope (TEM) images in Figure $S 2$ in the Supporting Information (SI)). We employ thiol surface chemistry to immobilize $\alpha$-Syn monolayer on the gold nanoantennas using a maleimide linker in TBS. First, a $\mathrm{COOH}$-functional alkanethiol SAM is formed on gold by 12 hours incubation in a solution of MHDA. Figure S3a shows the reflectance response of a nanorods array (L: $1850 \mathrm{~nm}$ and P: $2600 \mathrm{~nm}$ ) with SAM at different percentages of $\mathrm{MeOH}$ in buffer that is used as reference. The effect of water absorption signature (Figure S3b) from the aqueous background overlapping the amide I fingerprint is observed while the solution is changed from $40 \%$ to $0 \% \mathrm{MeOH}$ in TBS with the fluidic device. In addition, the methanol signatures in the $\sim 1400-1500 \mathrm{~cm}^{-1}$ range disappear in the reflectance responses upon decrease in $\mathrm{MeOH}$ concentration. The antenna design according to the slight blue shift of the far-field resonance peak is compared to its plasmonic near-field peak to provide maximum field enhancement at the amide I range. ${ }^{13}$ Therefore, as a rule of thumb, by using the antenna array with reflectance peak tuned toward the upper range of the amide I signature, we can provide optimum enhancement. As previously shown, approximately 3 orders of magnitude signal enhancement has been achieved with similar mid-IR nanorod antennas. ${ }^{14,27} \mathrm{Next}$, ex situ immobilization of protein via linker is performed using the surface functionalization steps explained in the Experimental Section. Here, we use a $12 \mu \mathrm{M} \alpha$-Syn stock solution that is freshly prepared in $40 \% \mathrm{MeOH}$ in TBS to immobilize the proteins on the gold antennas within an hour of incubation after surface functionalization. Finally, the substrate is rinsed to remove any excessive unbound protein and is measured in the fluidic device with the corresponding solution. The reflectance response of the antenna array with protein is measured at different conditions where the $\mathrm{MeOH}$ percentage is changed from $40 \%$ to $0 \%$ in sequential steps throughout the experiment as shown in Figure $2 \mathrm{a}$ and explained in the following. To extract
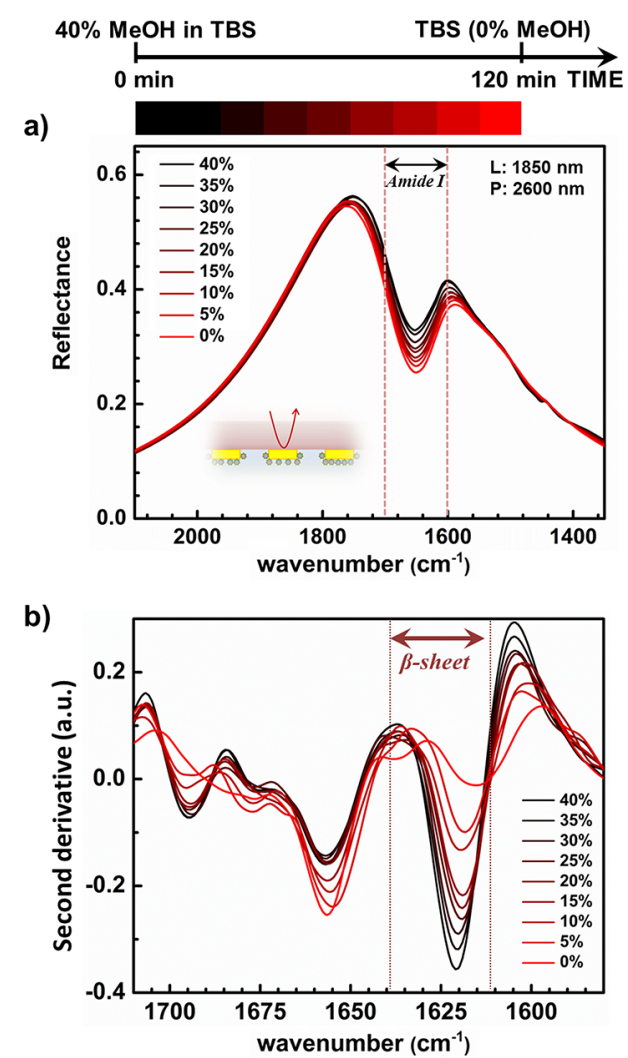

Figure 2. Real-time secondary structure detection and monitoring on immobilized $\alpha$-Syn monolayer. (a) Reflectance response of antenna arrays (L: $1850 \mathrm{~nm}$ and P: $2600 \mathrm{~nm}$ ) with protein monolayer. The methanol content in TBS is gradually changed from $40 \%$ to $0 \%$ over a period of $120 \mathrm{~min}$ in nine steps while the reflectance is acquired at every $15 \mathrm{~min}$. The amide I vibration range $\left(1600-1700 \mathrm{~cm}^{-1}\right)$ is marked by dashed lines. The schematic inset illustrates the plasmonic internal reflection measurement configuration that is employed with the integrated fluidic device. (b) Second derivative analysis of acquired amide I signature for the $\alpha$-Syn monolayer during buffer change steps calculated from the logarithmic ratio of reflectance responses with protein to without protein. The $\beta$-sheet component in the amide I fingerprint weakens upon gradual decrease of $\mathrm{MeOH}$ concentration while the disordered signature increases. The red arrow indicates the approximate range of $\beta$-sheet component.

the secondary structure components of $\alpha$-Syn within its amide I signature at each step, a second derivative of the acquired data with the logarithmic ratio of the reflectance responses $(A)$ is obtained using the reference measurement and is presented in Figure $2 b{ }^{22}$ First, the reflectance response is measured while the protein is initially exposed to $40 \% \mathrm{MeOH}$ in TBS in the fluidic channel after $1 \mathrm{~h}$ post-incubation wait (black curve in Figure 2a). Strong $\beta$-sheet signatures are observed in the 
1615-1640 $\mathrm{cm}^{-1}$ range with weaker signatures of this secondary structure at $\sim 1685-1695 \mathrm{~cm}^{-1}$ range (black curve in Figure 2b). We then flow reduced concentration of $\mathrm{MeOH}$ and acquire the reflectance response at every $15 \mathrm{~min}$ as presented in Figure 2a. The flow rate used for each step is 250 $\mu \mathrm{L} / \mathrm{min}$ during $6 \mathrm{~min}$ and the flow is stopped for $5 \mathrm{~min}$ before each reflectance measurement while the protein monolayer is exposed to the corresponding specified solution. Acquired results presented in Figure $2 \mathrm{~b}$ indicate that the $\alpha$-Syn monolayer, which was initially prepared in $40 \% \mathrm{MeOH}$ in TBS and immobilized under the same buffer condition, maintains its distinct $\beta$-sheet signature after immobilization and initial measurement in the same solution. Upon gradual removal of $\mathrm{MeOH}$ in the TBS surrounding the protein layer, the $\beta$-sheet signature weakens as expected while the component signature of disordered increases. However, once the methanol is completely removed from the buffer $(0 \% \mathrm{MeOH}$ in TBS, red curve in Figure 2a,b), the protein layer still incorporates some weak residual $\beta$-sheet signature at lower wavenumbers when measured after limited rest in TBS. In addition, more distinct features corresponding to non- $\beta$-sheet strands (turns and loops) are observed at this state $\left(\sim 1660-1685 \mathrm{~cm}^{-1}\right)$. Integration methods can be applied to present these conformational dynamics and variations as shown in Figure S4.

Analysis of Protein Secondary Structure Response to Stimuli. Our biosensor integrated with microfluidics facilitates introducing and switching between different solutions condition, and therefore provides great flexibility in modulating the structure of the immobilized proteins. In contrast to the static measurements with conventional bulk measurement methods such as circular dichroism spectroscopy, our approach can enable dynamic and comparative monitoring of reversible and irreversible conformational changes induced by stimuli. To demonstrate such capabilities, we examine the secondary structure changes of $\alpha$-Syn monolayer in two different experiments in which the influence of a denaturing agent is investigated when it is included within a full cycle of $\mathrm{MeOH}$ concentration change, as presented in Figure 3. Both experiments are conducted using identical arrays (L: 1850 $\mathrm{nm}$ and P: $2600 \mathrm{~nm}$ ) and immobilization procedures as presented before. The gradual concentration changes of $\mathrm{MeOH}$ in TBS from $40 \%$ to $0 \%$ and back to $40 \%$ are presented for decreasing (i.e., (i)) and increasing concentration (i.e., (ii)) parts for each of the two experiments. In the first experiment (Figure 3a), the $\alpha$-Syn monolayer is exposed to a high flow of TBS after methanol removal before being measured again in buffer at the end of part (i), whereas in the second experiment (Figure $3 \mathrm{~b}$ ) it is exposed to a denaturing agent at this step before being measured again in TBS as explained below.

In Figure $3 \mathrm{a}$ at the beginning of the first experiment, we similarly observe the initial decrease in $\beta$-sheet signature when $\mathrm{MeOH}$ concentration is decreased from $40 \%$ to $0 \%$ within 120 min in part (i) as schematically illustrated. At this step, we employ a TBS flow of $500 \mu \mathrm{L} / \mathrm{min}$ for $2.5 \mathrm{~min}$ which is then paused before measuring the protein again in TBS (i.e, at 135 min) shown with the last red curve in part (i) of Figure $3 a$. The results indicate that the weak residual $\beta$-sheet signature of the immobilized protein layer does not disappear even when it is exposed to TBS for more than $15 \mathrm{~min}$. Next, we continue the experiment by progressively increasing the $\mathrm{MeOH}$ concentration from $0 \%$ to $40 \%$ in part (ii) of Figure 3 a with similar flow rates and timing as in previous experiments to complete a full cycle of buffer change. Here, the first red curve in part (ii) is a)
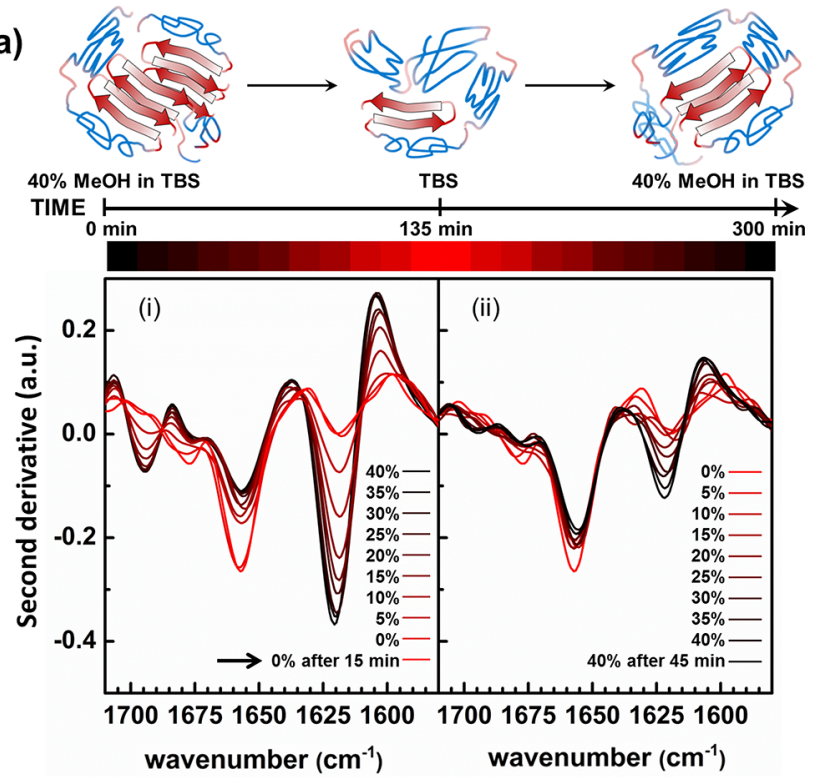

b)
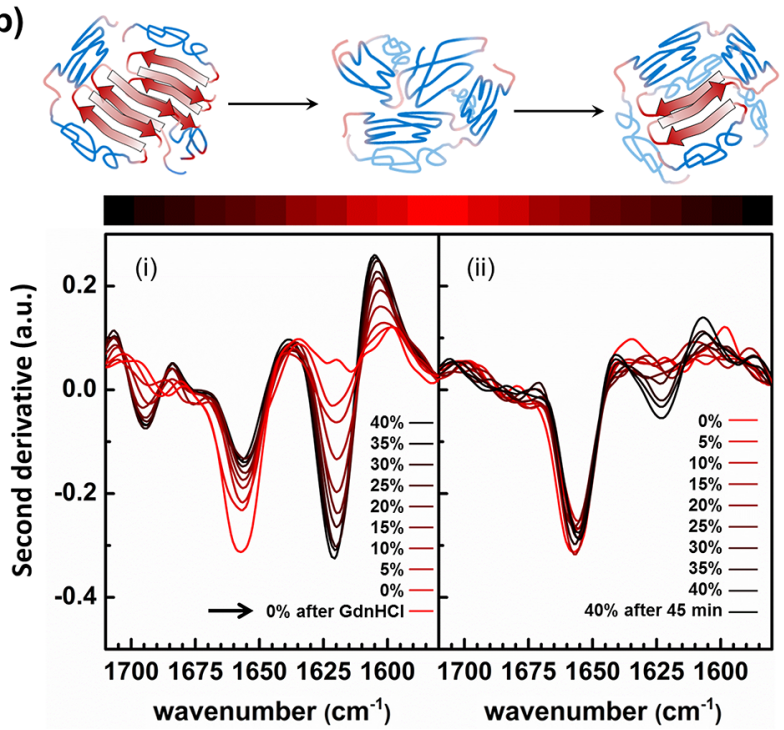

Figure 3. Secondary structure analysis on $\alpha$-Syn monolayer with nanoplasmonics during solution change cycles in two experiments with and without denaturing agent $(\mathrm{GdnHCl})$ and the corresponding schematic illustrations. (a) Immobilized $\alpha$-Syn secondary structural changes induced with varying $\mathrm{MeOH}$ concentration only: (i) response to $\mathrm{MeOH}$ concentration change from $40 \%$ to $0 \%$ in TBS (by 120 $\mathrm{min})$. At the final stage, a high flow of TBS is used before measuring the response at TBS again (i.e., $135 \mathrm{~min}$ ). The residual $\beta$-sheet signature is detected as depicted in the top illustration. (ii) Response to increase of $\mathrm{MeOH}$ concentration back to $40 \%$ in TBS (i.e., at 255 $\mathrm{min}$ ). At the final stage the monolayer is measured after waiting 45 min in $40 \% \mathrm{MeOH}$ in TBS at the end of a full cycle. (b) Immobilized $\alpha$-Syn secondary structural changes induced with methanol concentration and denaturant: (i) response to the change from $40 \% \mathrm{MeOH}$ in TBS to TBS within 120 min. At the final stage in part (i), GdnHCl in TBS is introduced in the fluidic device for $2.5 \mathrm{~min}$ before changing the solution back to TBS and measuring the monolayer again (i.e., 135 min). (ii) Response to increase of $\mathrm{MeOH}$ concentration back to $40 \%$ (i.e., at $255 \mathrm{~min}$ ). At the final stage the monolayer is measured after waiting $45 \mathrm{~min}$ in $40 \% \mathrm{MeOH}$ in TBS. The last curves in each part (i) are shown again in their corresponding next parts (i.e., (ii)) for easier monitoring of both experiments in (a) and (b).

the last red curve in part (i) corresponding to protein at TBS with no $\mathrm{MeOH}$. Comparing the $\beta$-sheet signature of the $\alpha$-Syn 
monolayer in part (i) and (ii) for the same $\mathrm{MeOH}$ concentration indicate significantly lower $\beta$-sheet signals during increasing methanol percentage as opposed to the previous steps with decreasing methanol. This observation could be as a result of several factors such as immobilization of the protein on the gold surface that could affect the formation of $\beta$-sheet, the protein's previous conformational state at each step or the time needed for immobilized proteins to react to the environmental changes. At the final step in this cycle, the black curve in part (ii), we measure the response of the protein layer in $40 \% \mathrm{MeOH}$ after waiting an additional $45 \mathrm{~min}$ in the solution with no flow. Here, we observe a slight increase in the $\beta$-sheet signature when the $\alpha$-Syn layer is exposed to the $\mathrm{MeOH}$ solution for longer time.

Similarly, we performed the second experiment with identical timing for the experimental steps but use the chemical denaturant guanidine hydrochloride $(\mathrm{GdnHCl})$ to unfold the protein. The results for this experiment are presented in Figure 3b where in part (i) the $\mathrm{MeOH}$ concentration is similarly reduced from $40 \%$ to $0 \%$. The initial results in part (i) reproduce our previous experiments in Figure $3 a$ and Figure $2 b$. In the final steps of part (i) when TBS with no $\mathrm{MeOH}$ is in the flow channel, we employ a flow rinse step with $6 \mathrm{M} \mathrm{GdnHCl}$ in TBS for 2.5 min followed by TBS flow before measuring the protein in buffer again (at $135 \mathrm{~min}$ ). As schematically illustrated, the denaturant properties of $\mathrm{GdnHCl}$ should remove any residual structure in the proteins and result in random coiled structure of $\alpha$-Syn layer after its exposure which is observed in the last red curve in part (i) of Figure $3 \mathrm{~b}$ measured in TBS. Continuing the rest of this experiment analogous to the previous one results in observing a similar trend. In addition, the two experiments show that the residual $\beta$-sheet conformation at the final step in part (i) (Figure 3a) contributes to slightly higher final $\beta$-sheet signatures when comparing the final results of part (ii) in Figure $3 a$ and $b$. This is in line with the observation that after total removal of the $\mathrm{MeOH}$ and its reintroduction, the protein layer does not form as much $\beta$-sheet compared to its initial state. In addition, we conducted an experiment with an identical array and preparation steps in which we initially immobilized $\alpha$-Syn protein prepared in TBS on the antenna surface. The results for this test are shown in Figure S5 where after immobilization and measurement of the protein monolayer at its initial state the solution is changed by directly flowing $40 \% \mathrm{MeOH}$ in TBS with the fluidic device. In this experiment the appearance of $\beta$ sheet signatures due to presence of high percentage of $\mathrm{MeOH}$ in the buffer is dramatically slower compared to the previous tests. Moreover, the weak $\beta$-sheet signature observed in the protein monolayer after $4 \mathrm{~h}$ of exposure to $40 \% \mathrm{MeOH}$ appears at slightly higher wavenumbers $\left(\sim 5 \mathrm{~cm}^{-1}\right)$ compared to that of the previous experiments. These results can indicate that the protein conformational response to stimuli and environmental changes is influenced by immobilization and its initial conformational state in addition to the timing of introducing such triggers.

Comparison of Nanoplasmonic Method with Infrared Transmission and Grazing Incidence Reflection. The exact peak frequency of each secondary structural component within the amide I vibrational band is important for interpreting protein conformational states and analyzing different proteins. $^{20,22}$ Therefore, we validated the results extracted from our new nanoplasmonics approach with two conventional IR spectroscopy techniques, namely, infrared transmission and grazing incidence reflection (GIR) configurations. Transmission measurements are the general method for evaluating thick films and large sample volumes whereas GIR measurements enable infrared reflection-absorption spectroscopy of thin films on gold surface and therefore resemble our approach. ${ }^{21,36}$ For this comparison, we use PMMA (poly(methyl methacrylate)) polymer and experimentally analyzed its distinct carbonyl signature with these three techniques without any overlapping background signals in dry condition. The schematic illustrations in Figure 4a depict the two different
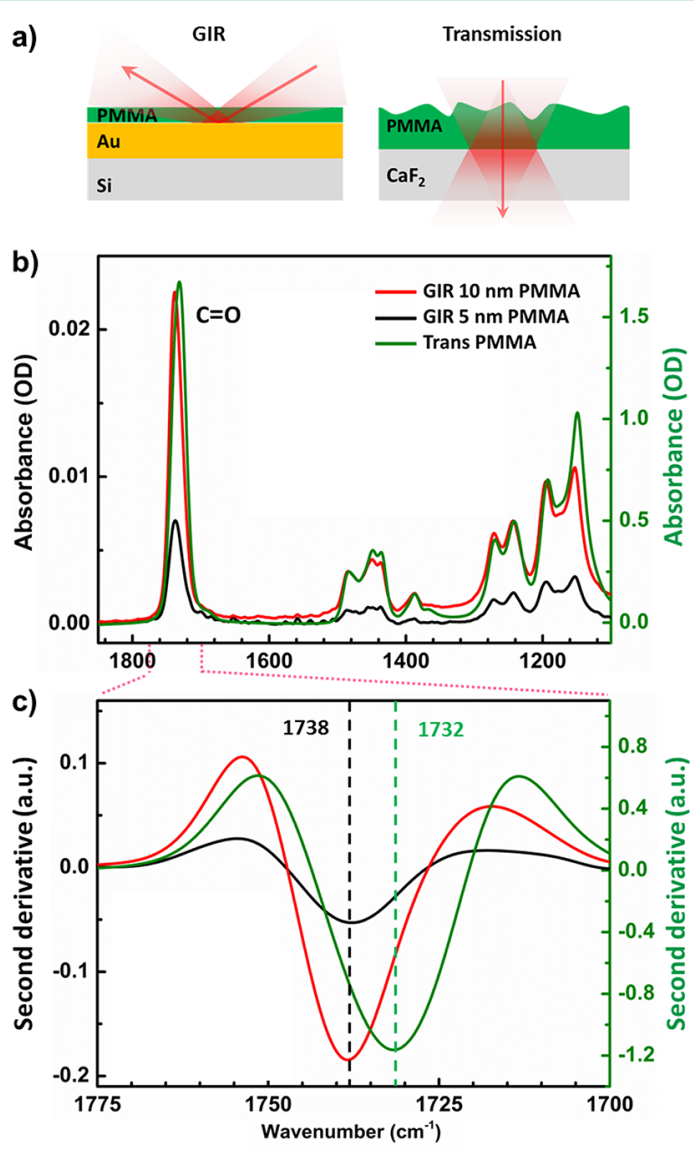

Figure 4. Extracting CO vibrational component of PMMA films with IR transmission and grazing incidence reflection measurements through second derivative analysis. (a) Schematic illustrations of (left) grazing incidence reflection (GIR) measurement of thin PMMA films on $\mathrm{Au}$ and (right) IR transmission measurements of dried PMMA layer on $\mathrm{CaF}_{2}$ substrate. (b) Absorbance spectra of dropcasted PMMA layer measured with IR transmission (green curve, right axis) and spin-coated thin films ( $5 \mathrm{~nm}$, black curve; and $10 \mathrm{~nm}$, red curve; left axis) measured with GIR configuration. The distinct $\mathrm{CO}$ vibrational signature at $\sim 1700-1770 \mathrm{~cm}^{-1}$ is observed. (c) Second derivative analysis of the $\mathrm{CO}$ vibration signature from (b) and the corresponding extracted component frequency with the two measurement configurations.

measurements for transmission and GIR configuration both performed with an IR microscope. For transmission measurement, we dropcast a thick PMMA (950 MW) layer on a clean $\mathrm{CaF}_{2}$ substrate and measure its transmission after 2 min drying on a hot plate. The resulting absorbance from this measurement is shown in Figure $4 \mathrm{~b}$ with the green curve. For GIR measurements, we used a grazing incidence objective with 52$84^{\circ}$ angle of incidence. In this case, the thin PMMA films on gold substrates are prepared with spin coating. Two different 
thickness of 5 and $10 \mathrm{~nm}$ are analyzed, representing typical thicknesses of immobilized protein layers in bioassays. The thin film signatures are extracted with GIR measurements using $\alpha=$ $-\log \left(R_{\text {with film }} / R_{\text {bare }}\right)$ and are presented in Figure $4 \mathrm{~b}$. The corresponding results show the different absorption signals of the PMMA layers with the two measurement configurations. Here, the strength of the absorbance is not relevant for comparing the resulted peak frequency of an acquired vibrational component. Multiple vibrational signatures of the PMMA layer can be observed in Figure $4 \mathrm{~b}$. Notably, the distinct $\mathrm{CO}$ vibration without any overlapping background signals allows experimental assessment for the exact frequency of this individual component while avoiding variations due to presence of any neighboring spectral components. In Figure 4c, we compare the acquired frequency of $\mathrm{CO}$ vibration from the two different measurement approaches by using the second derivative analysis within this vibrational band. Here, we observe the well-known slight blue shift between the transmission $\left(\sim 1732 \mathrm{~cm}^{-1}\right)$ and GIR $\left(\sim 1738 \mathrm{~cm}^{-1}\right)$ results which corresponds to anomalous dispersion of the refractive index as shown in literature. ${ }^{36,37}$ Next, we evaluate our nanoplasmonic approach by fabricating two sets of antenna arrays with varying $\mathrm{L}$ and $\mathrm{P}$ in order to tune the resonance peak throughout the carbonyl vibration and provide different full width half maximums (fwhm) of resonances. The reflectance responses are shown in Figure $5 b$, for bare arrays. The antenna designs are L: 2000, 2050, 2100, and $2150 \mathrm{~nm}$ with P: $3 \mu \mathrm{m}$ (solid curves) and L: 2000, 2050, 2100, 2150, and $2200 \mathrm{~nm}$ with P: $2.5 \mu \mathrm{m}$ (dashed curves). The gray shaded strip indicates the approximate frequency of the PMMA carbonyl signature. By sweeping the peak and bandwidth of resonances, we can provide a comprehensive investigation on the influence of resonance excitation and coupling conditions for detecting vibrational components with our nanoplasmonic method in relation to other IR techniques. Two identical substrates were spin coated with thin PMMA layers similar to that of GIR samples (i.e., 5 and $10 \mathrm{~nm}$ ) and the reflectance response was measured as illustrated in Figure 5a. The reflectance spectra are used to extract the frequency of vibrational signature with second derivative analysis of the logarithmic ratio of the reflectance responses $(A)$ before and after coating as introduced previously. The acquired frequency results of the carbonyl component for the two PMMA thicknesses are presented in Figure $5 \mathrm{c}$ as data points corresponding to the peak of the extracted vibrational component. The dashed lines correspond to results extracted from Figure $4 \mathrm{c}$ using infrared transmission (green) and grazing incident reflection (black) measurements. Figure S6 shows the second derivative analysis for some of the arrays from which the data points in Figure $5 \mathrm{c}$ are retrieved as the peak of the vibrational component. Even with the detuning of the antenna array responses over a wide range across the CO vibration, the acquired data points are consistently clustered and exhibit only a $4 \mathrm{~cm}^{-1}$ spread for the thin films. Based on these experimental results, the nanoplasmonics approach is interestingly in-line with IR transmission. The results establish the reliability of our surface-based detection approach for analyzing thin samples relevant to biological applications.

\section{CONCLUSION}

We experimentally demonstrated the unique capabilities for ultrasensitive real-time secondary structure detection on a protein monolayer and thin films using mid-IR nanoplasmonic antennas. Our biosensing platform enables reliable monitoring
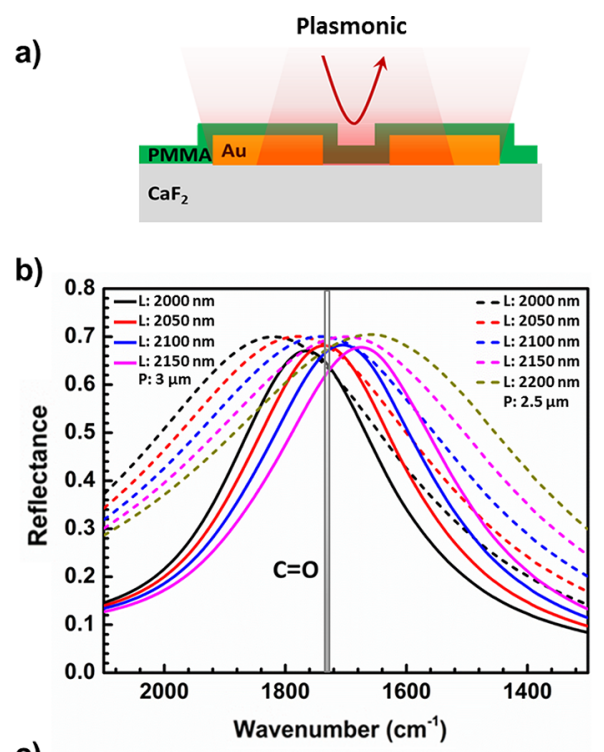

c)

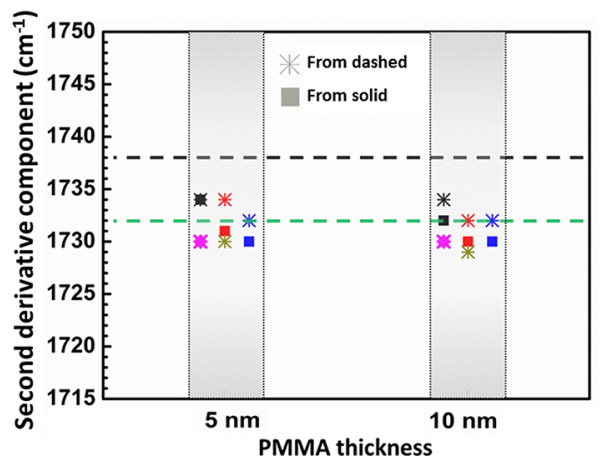

Figure 5. Extracting CO vibrational component of polymer thin films with mid-IR nanoplasmonic antenna arrays through second derivative analysis. (a) Schematic illustrations of reflectance measurements of dried PMMA thin film on antennas. (b) Reflectance response of bare antenna arrays L: 2000, 2050, 2100, and $2150 \mathrm{~nm}$ with P: $3 \mu \mathrm{m}$ (solid) and L: 2000, 2050, 2100, 2150, and $2200 \mathrm{~nm}$ with P: $2.5 \mu \mathrm{m}$ (dashed). Gray vertical strip shows the approximate frequency of the $\mathrm{CO}$ vibration. (c) Extracted frequency of the $\mathrm{CO}$ component for 5 and 10 $\mathrm{nm}$ thin PMMA spin-coated on identical plasmonic substrates with arrays shown in (b). Colored data points are acquired through second derivative analysis of the plasmonically enhanced vibrational signature of PMMA from each of the antenna arrays presented in (b) with the same color. The dashed lines correspond to the results of this component acquired from the transmission (green) and GIR (black) measurements shown in Figure 4.

of protein conformational changes under various conditions through the amide I signature as it is the most prominent vibrational band of all proteins. To validate that our sensor can reliably acquire the component frequencies of vibrational signatures of thin films, we compared our approach with two conventional IR spectroscopy techniques and demonstrated its correlation to IR transmission results. Our surface-based nanosensor is promising for mid-IR secondary structural analysis on minute protein samples including transient species in dynamic conformational processes, such as amyloidogenic proteins during fibrillization. The nanostructures are made out of gold which allows exploitation of well-established and biocompatible surface functionalization approaches for monitoring biomolecular interactions with a wide range of stimuli including lipids, enzymes, peptides, or environmental con- 
ditions such as $\mathrm{pH}$, temperature, or ionic strength changes toward mimicking interactions at the cell membrane. To minimize interference from surface immobilization and retain the native functionality of the proteins, advanced surface chemistry and molecular biology strategies can be employed, for instance, by introducing specific functional groups in regions outside the domains of interest or in flexible parts of the proteins. Additionally, the response of the nanoantennas can be engineered to enable access of other important vibrational bands in proteins (e.g., amides II and III) for a more comprehensive IR analysis of samples. Employing normalincidence reflectance measurements with this ultrasensitive detection principle can facilitate implementation of imaging techniques and therefore multiplexing capabilities. In contrast to conventional IR methods, this nanoplasmonic sensor can offer numerous potentials for integration with chip-based midIR technologies in a compact device footprint in conjunction with advanced microfluidics for automated and dynamic study of protein conformations. Providing such a platform to analyze, monitor, and potentially quantify protein secondary changes under physiological conditions offers new opportunities to investigate the conformational properties of biologically relevant species and develop novel assays to identify inhibitors that target the early steps of protein misfolding in the pathways to forming pathological aggregates.

\section{ASSOCIATED CONTENT}

\section{(5) Supporting Information}

The Supporting Information is available free of charge on the ACS Publications website at DOI: 10.1021/acssensors.8b00115.

Bulk CD characterization of $\alpha$-synuclein, TEM analysis of $\alpha$-synuclein in $40 \%$ methanol, Reflectance response of nanorods array with SAM in solution, Integrated $\beta$-sheet signals extracted from Figure $2 \mathrm{~b}$, Immobilization of $\alpha$ Syn protein in TBS on the antenna arrays, Example of second derivative analysis for four arrays presented in Figure 5b. (PDF)

\section{AUTHOR INFORMATION}

\section{Corresponding Author}

*E-mail: hatice.altug@epfl.ch.

\section{ORCID 1}

Dordaneh Etezadi: 0000-0003-4289-2476

John B. Warner IV: 0000-0001-5562-5620

Hilal A. Lashuel: 0000-0001-7682-8320

\section{Notes}

The authors declare no competing financial interest.

\section{ACKNOWLEDGMENTS}

We acknowledge École Polytechnique Fédérale de Lausanne (EPFL) and Center of MicroNano Technology (CMi) for financial support and nanofabrication. The research leading to these results has received funding from European Research Council (ERC) under grant agreement no. 682167 VIBRANTBIO. We also thank European Union Horizon 2020 Framework Programme for Research and Innovation, under grant agreements no. 777714 (NOCTURNO project) and no. FETOPEN-737071 (ULTRACHIRAL project).

\section{REFERENCES}

(1) Mayer, K. M.; Hafner, J. H. Localized surface plasmon resonance sensors. Chem. Rev. 2011, 111 (6), 3828-57.

(2) McLeod, E.; Ozcan, A. Unconventional methods of imaging: computational microscopy and compact implementations. Rep. Prog. Phys. 2016, 79 (7), 076001-30.

(3) Sriram, M.; Zong, K.; Vivekchand, S. R.; Gooding, J. J. Single nanoparticle plasmonic sensors. Sensors 2015, 15 (10), 25774-92.

(4) Hill, R. T. Plasmonic biosensors. Wiley Interdisciplinary Reviews: Nanomedicine and Nanobiotechnology. 2015, 7 (2), 152-68.

(5) Lopez, G. A.; Estevez, M. C.; Soler, M.; Lechuga, L. M. Recent advances in nanoplasmonic biosensors: Applications and lab-on-a-chip integration. Nanophotonics 2017, 6 (1), 123-36.

(6) Wu, Z.; Kelp, G.; Yogeesh, M. N.; Li, W.; McNicholas, K. M.; Briggs, A.; Rajeeva, B. B.; Akinwande, D.; Bank, S. R.; Shvets, G.; Zheng, Y. Dual-band moiré metasurface patches for multifunctional biomedical applications. Nanoscale 2016, 8 (43), 18461-8.

(7) Hong, K. Y.; de Albuquerque, C. D.; Poppi, R. J.; Brolo, A. G. Determination of Aqueous Antibiotic Solutions Using SERS Nanogratings. Anal. Chim. Acta 2017, 982, 148-155.

(8) Halas, N. J.; Lal, S.; Chang, W.-S.; Link, S.; Nordlander, P. Plasmons in strongly coupled metallic nanostructures. Chem. Rev. 2011, 111, 3913-3961.

(9) Rodrigo, D.; Limaj, O.; Janner, D.; Etezadi, D.; Garcia de Abajo, F. J.; Pruneri, V.; Altug, H. Mid-infrared plasmonic biosensing with graphene. Science 2015, 349 (6244), 165-8.

(10) Hoffmann, J. M.; Yin, X.; Richter, J.; Hartung, A.; Maß, T. W.; Taubner, T. Low-cost infrared resonant structures for surfaceenhanced infrared absorption spectroscopy in the fingerprint region from 3 to $13 \mu \mathrm{m}$. J. Phys. Chem. C 2013, 117 (21), 11311-6.

(11) Hu, H.; Yang, X.; Zhai, F.; Hu, D.; Liu, R.; Liu, K.; Sun, Z.; Dai, Q. Far-field nanoscale infrared spectroscopy of vibrational fingerprints of molecules with graphene plasmons. Nat. Commun. 2016, 7, 123348.

(12) Chen, K.; Dao, T. D.; Nagao, T. Tunable Nanoantennas for Surface Enhanced Infrared Absorption Spectroscopy by Colloidal Lithography and Post-Fabrication Etching. Sci. Rep. 2017, 7, 44069-8.

(13) Neubrech, F.; Huck, C.; Weber, K.; Pucci, A.; Giessen, H. Surface-Enhanced Infrared Spectroscopy Using Resonant Nanoantennas. Chem. Rev. 2017, 117 (7), 5110-45.

(14) Adato, R.; Altug, H. In-situ ultra-sensitive infrared absorption spectroscopy of biomolecule interactions in real time with plasmonic nanoantennas. Nat. Commun. 2013, 4, 2154-2163.

(15) Dong, L.; Yang, X.; Zhang, C.; Cerjan, B.; Zhou, L.; Tseng, M. L.; Zhang, Y.; Alabastri, A.; Nordlander, P.; Halas, N. J. 2017. Nanogapped Au Antennas for Ultrasensitive Surface-Enhanced Infrared Absorption Spectroscopy. Nano Lett. 2017, 17 (9), 57685774.

(16) Ataka, K.; Kottke, T.; Heberle, J. Thinner, smaller, faster: IR techniques to probe the functionality of biological and biomimetic systems. Angew. Chem., Int. Ed. 2010, 49, 5416-5424.

(17) Kendall, C.; Isabelle, M.; Bazant-Hegemark, F.; Hutchings, J.; Orr, L.; Babrah, J.; Baker, R.; Stone, N. Vibrational spectroscopy: a clinical tool for cancer diagnostics. Analyst 2009, 134 (6), 1029-45.

(18) Nabers, A.; Ollesch, J.; Schartner, J.; Kötting, C.; Genius, J.; Haußmann, U.; Klafki, H.; Wiltfang, J.; Gerwert, K. An infrared sensor analysing label-free the secondary structure of the Abeta peptide in presence of complex fluids. Journal of biophotonics. 2016, 9 (3), 22434.

(19) Wehbe, K.; Pinneau, R.; Moenner, M.; Déléris, G.; Petibois, C. FT-IR spectral imaging of blood vessels reveals protein secondary structure deviations induced by tumor growth. Anal. Bioanal. Chem. 2008, 392 (1-2), 129-35.

(20) Fabian, H.; Naumann, D. Protein Folding and Misfolding: Shining Light by Infrared Spectroscopy; Springer: Heidelberg, 2012.

(21) Yang, H.; Yang, S.; Kong, J.; Dong, A.; Yu, S. Obtaining information about protein secondary structures in aqueous solution using Fourier transform IR spectroscopy. Nat. Protoc. 2015, 10, 382396. 
(22) Dong, A.; Huang, P.; Caughey, W. S. Protein secondary structures in water from second-derivative amide I infrared spectra. Biochemistry 1990, 29, 3303-3308.

(23) Schwaighofer, A.; Alcaráz, M. R.; Araman, C.; Goicoechea, H.; Lendl, B. External cavity-quantum cascade laser infrared spectroscopy for secondary structure analysis of proteins at low concentrations. Sci. Rep. 2016, 6, 33556-66.

(24) Soto, C.; Estrada, L.; Castilla, J. Amyloids, prions and the inherent infectious nature of misfolded protein aggregates. Trends Biochem. Sci. 2006, 31, 150-155.

(25) Knowles, T. P. J.; Vendruscolo, M.; Dobson, C. M. The amyloid state and its association with protein misfolding diseases. Nat. Rev. Mol. Cell Biol. 2014, 15, 384-396.

(26) Greenfield, N. J. Using circular dichroism spectra to estimate protein secondary structure. Nat. Protoc. 2007, 1, 2876-2890.

(27) Etezadi, D.; Warner, J. B., IV; Ruggeri, F. S.; Dietler, G.; Lashuel, H. A.; Altug, H. Nanoplasmonic mid-infrared biosensor for in vitro protein secondary structure detection. Light: Sci. Appl. 2017, 6 (8), e17029-39.

(28) Lashuel, H. A.; Overk, C. R.; Oueslati, A.; Masliah, E. The many faces of $\alpha$-synuclein: from structure and toxicity to therapeutic target. Nat. Rev. Neurosci. 2013, 14, 38-48.

(29) Bodner, C. R.; Maltsev, A. S.; Dobson, C. M.; Bax, A. Differential phospholipid binding of $\alpha$-synuclein variants implicated in Parkinson's disease revealed by solution NMR spectroscopy. Biochemistry 2010, 49 (5), 862-71.

(30) Bodner, C. R.; Dobson, C. M.; Bax, A. Multiple tight phospholipid-binding modes of $\alpha$-synuclein revealed by solution NMR spectroscopy. J. Mol. Biol. 2009, 390 (4), 775-90.

(31) Munishkina, L. A.; Phelan, C.; Uversky, V. N.; Fink, A. L. Conformational behavior and aggregation of $\alpha$-synuclein in organic solvents: modeling the effects of membranes. Biochemistry 2003, 42 (9), 2720-30.

(32) Adato, R.; Yanik, A. A.; Amsden, J. J.; Kaplan, D. L.; Omenetto, F. G.; et al. Ultra-sensitive vibrational spectroscopy of protein monolayers with plasmonic nanoantenna arrays. Proc. Natl. Acad. Sci. U. S. A. 2009, 106, 19227-19232.

(33) Neubrech, F.; Pucci, A.; Cornelius, T. W.; Karim, S.; GarcíaEtxarri, A.; et al. Resonant plasmonic and vibrational coupling in a tailored nanoantenna for Infrared detection. Phys. Rev. Lett. 2008, 101, 157403-4.

(34) Aksu, S.; Yanik, A. A.; Adato, R.; Artar, A.; Huang, M.; Altug, H. High-throughput nanofabrication of infrared plasmonic nanoantenna arrays for vibrational nanospectroscopy. Nano Lett. 2010, 10 (7), 2511-8.

(35) Limaj, O.; Etezadi, D.; Wittenberg, N. J.; Rodrigo, D.; Yoo, D.; Oh, S. H.; Altug, H. Infrared plasmonic biosensor for real-time and label-free monitoring of lipid membranes. Nano Lett. 2016, 16 (2), $1502-8$.

(36) Mastel, S.; Govyadinov, A. A.; de Oliveira, T. V.; Amenabar, I.; Hillenbrand, R. Nanoscale-resolved chemical identification of thin organic films using infrared near-field spectroscopy and standard Fourier transform infrared references. Appl. Phys. Lett. 2015, 106 (2), 023113-5.

(37) Yamamoto, K.; Ishida, H. Optical theory applied to infrared spectroscopy. Vib. Spectrosc. 1994, 8 (1), 1-36. 